\title{
Image and audio fractal compression: comprehensive review, enhancements and research directions
}

\author{
Ahmed Hussain Ali ${ }^{1}$, Ali Nihad Abbas ${ }^{2}$, Loay Edwar George ${ }^{3}$, Mohd Rosmadi Mokhtar ${ }^{4}$ \\ ${ }^{1,4}$ Universiti Kebangsaan Malaysia, Malaysia \\ ${ }^{1,2}$ Ministry of Higher Education and Scientific Research, Studies Planning and Follow-up Directorate, Iraq \\ ${ }^{3}$ University of Baghdad, Iraq
}

\section{Article Info}

Article history:

Received Jan 17, 2019

Revised Apr 19, 2019

Accepted May 10, 2019

\section{Keywords:}

Compression

Fractal audio coding (FAC)

Fractal image coding (FIC)

\begin{abstract}
This study aims to review the recent techniques in digital multimedia compression with respect to fractal coding techniques. It covers the proposed fractal coding methods in audio and image domain and the techniques that were used for accelerating the encoding time process which is considered the main challenge in fractal compression. This study also presents the trends of the researcher's interests in applying fractal coding in image domain in comparison to the audio domain. This review opens directions for further researches in the area of fractal coding in audio compression and removes the obstacles that face its implementation in order to compare fractal audio with the renowned audio compression techniques.
\end{abstract}

Copyright $@ 2019$ Institute of Advanced Engineering and Science. All rights reserved.

Corresponding Author:

Ahmed Hussain Ali,

Universiti Kebangsaan Malaysia, Malaysia.

Email: ahmedhussainali78@gmail.com

\section{INTRODUCTION}

The development of digital multimedia and communication applications and the huge volume of data transfer through the internet place the necessity for data compression methods at the first priority [1]. The main purpose of data compression is to reduce the amount of transferred data by eliminating the redundant information to keep the transmission bandwidth and without significant effects on the quality of the data [2]. In addition, the compression time should be minimized. Image and audio are the most common medium used in communication between the end users along with the video. Many techniques are used to compress these multimedia files and they exhibit various degrees of compression rate and quality. The data compression is mainly classified into lossy and lossless compression techniques [3]. Lossless compression techniques have the advantage that the size of the original and the compressed data is the same. In addition, the reconstructed data is exactly the same as the original one [4]. However, the compression rate is normally lower than lossy techniques. There are several instances of type of compression techniques, RLE, Huffman, and arithmetic coding. In lossy compression, some of the data is lost during the compression process in order to reduce the size of the compressed file. Such techniques are widely used on the internet. DCT, DWT, VQ, and FC are examples of lossy compression techniques which can be applied to both image and audio files [5]. This study discusses the improvement on the fractal coding and shows the performance of applying it on image and audio files.

\section{FRACTAL THEORY}

The history of fractal compression belongs to the mathematician Benoit B. Mandelbrot who first introduced term fractal in the field of geometry in 1977 in his book entitled The Fractal Geometry of Nature. 
Later, this idea was applied to the image as a compression technique with the method of Iterated Function System (IFS) by Barnsley in 1988. In 1992 Arnode Jacquin finally used Partitioned Iterated Function System (PIFS) and collage theory in order to practically implement the fractal coding algorithm [6].

The basic idea behind FIC is to represent the image by a set of contractive transformations through exploiting the self-similarity in the image parts using PIFS. The encoding process consists of three subprocesses: first, partitioning the image into non-overlapped range and overlapped domain blocks, second, searching for similar range-domain with minimum error and third, storing the IFS coefficients in a file that represents a compress file and use it decompression process while the decoding process is a straightforward process [4]. Figure 1 and Figure 2 illustrate the two main processes.

FIC have several characteristics which are an asymmetric process, high compression ratio, good reconstructed image quality, and fast, straightforward decoding process. However, the encoding process is time-consuming since the exhaustive search process is required for complex computations [7, 8]. In literature, many techniques were presented to overcome the large encoding time.

It is worth to mention that many studies in literature presented enhancements in FIC, but few of them adopted the traditional fractal image model on the audio signal as shown in Section 6. This limitation is due to several obstacles that face the fractal image coding and thus made the FIC unpractical. In addition, there is the uncertainty of finding self-similarity in audio signals using the traditional fractal coding and the large size of the audio signal in comparison to images $[6,9]$. However, several techniques were adopted along with fractal coding to optimize the encoding time in the audio domain.

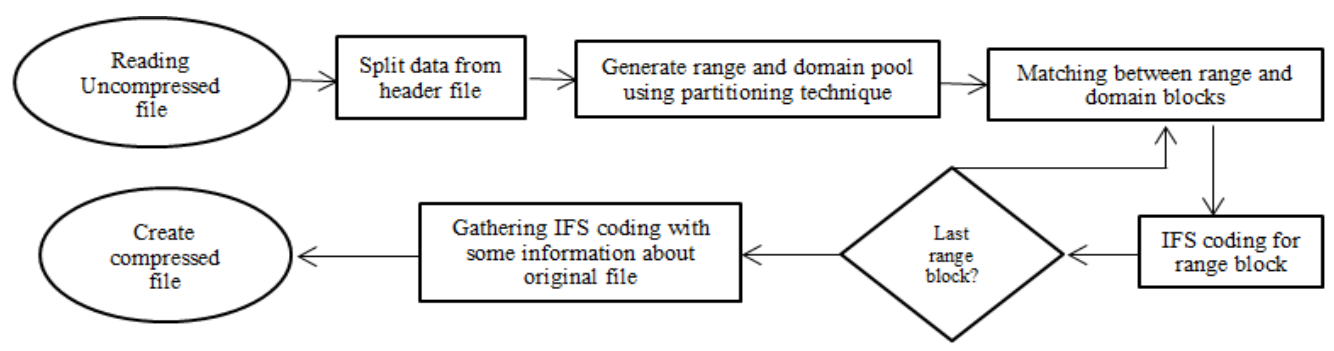

Figure 1. Fractal encoding

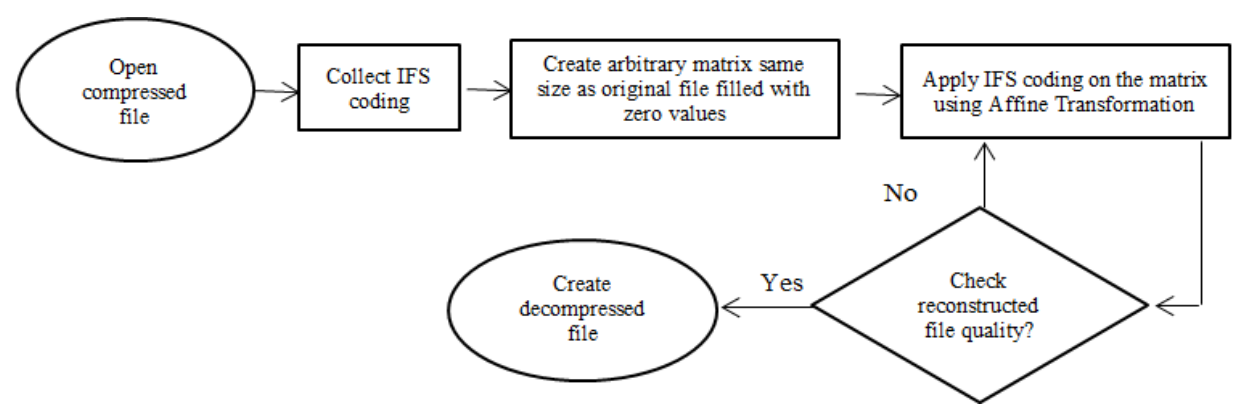

Figure 2. Fractal decoding

\section{FRACTAL DOMAINS}

Fractal domain means the media that is used to apply FC on. Generally, there are two main domains that should be considered when mentioning on FC in compression: image and audio. The literature showed that the fractal theory was firstly implemented on images by Fisher. The reason for choosing images was because the images exhibit a level of geometric feature. The gray image was a type of the images that were adopted to evaluate the efficiency of applying the fractal theory. These images are two-dimensional $\mathrm{M}^{*} \mathrm{~N}$ and uncompressed formatting matrix in which each pixel treated as a point with $\mathrm{x}$ and $\mathrm{y}$ coordinates. The blocks which are used in these experiments were of two dimensions with $2 \times 2,4 \times 4,16 \times 16$ pixels. Then several studies later used color images with 24-bit and results are investigated to show the effect of the FIC on it $[10,11]$.

The audio signal is adopted later by researchers and used with fractal theory. The reason for this adaptation was identical in audio and image data and the implementation on audio signal could produce the same impact. However, the effect is variant in both kinds of data because the Human Visual System (HVS)

Image and audio fractal compression: comprehensive review, enhancements and... (Ahmed Hussain Ali) 
and the Human Auditory System (HAS) are different [12]. The audio signal that is utilized in literature was uncompressed, one dimension signal with different sampling rate. The data is treated as a sequence of samples each one with 8-bit. The range and domain blocks also represent in 1-D with a different number of samples. A thorough investigation is required to check the ability to enhance the performance of the fractal audio compression.

\section{FRACTAL CODING CHALLENGES}

There are several common issues in the implementation of fractal coding in both image and audio files. These issues were considered challenges in adopting FC together with the popular compression technique nowadays. These challenges are motivation for further research in both domains and can be summarized as [4]:

a) Generating range and domain blocks using different partition methods.

b) Matching process between range-domain blocks in order to find the best similarity between then using measurement to locate the minimum distortion.

c) Reducing the encoding time by adopting speeding up techniques to minimize the time required.

d) Shrinking the domain pool to reduce the computation in the matching process.

\section{FRACTAL CODING ENHANCEMENTS}

Several ways have been suggested recently to solve a aforementioned issues in the FC model. The adaptation of various speeding up techniques was found more in images than audio domains. These developments can be classified mainly into these categories:

a) Optimization of pre-matching process

Searching or matching process is considered as the core of the fractal coding technique in which each range block matches with all blocks in the domain pool $[6,13]$. This process is a time-consuming process. Many techniques were proposed to reduce the matching process and reduce the number of the range and domain block as well. Partitioning, classification, and clustering are the most techniques used at this point. These techniques are considered as a pre-processing technique to filter out the domain blocks before the start in the matching process.

b) Parallel processing

Another way to reduce the large encoding time associated with FC is the parallel implementation. The development of the parallel computer architecture of the computational was the motivation to utilize this feature in accelerating the expensive complexity and then the efficiency of FC. Several studies were proposed different techniques of parallel computing on FIC ranging from complexity reduction, load balance and data partitioning depending on the proposed algorithm and hardware platforms. However, interest readers can follow [14] for more details about parallel algorithms in Fractal Image Compression.

c) Neural network

Soft computing methods are generally used to solve the problems that cannot be solved by traditional ones or that ones that take a long time. The large FC encoding time can be optimized using unconventional methods. Neural network and genetic algorithm were two of the nominated methods to settle consuming time issues. Several researches were proposed in this sense with different level of performances. In the last decades, FIC was the target of the researchers for adopting neural network and genetic algorithms to enhance efficiency. The analysis of the proposed FIC methods presented in [9-11] show that the performance of adopting this type of techniques with FIC in term of compression ratio is lower in comparison to the common method.

\section{LITERATURE REVIEW}

Fractal image and audio compression methods are reviewed in this section. The techniques that proposed along with the traditional fractal coding for enhancing the performance are also presented. |The focus is on the first category of the enhancements that were proposed in the literature which is related to the optimization of the pre-matching process in Section 5. Table 1 summarizes the below literature review studies.

\subsection{Fractal Image Compression}

The interest in FIC belongs to 1988 when Barnsley applied IFS to images for compression. Several methods were adopted since 2000 . 
Truong et al. [15] proposed an algorithm to reduce the redundant calculation of the inner product in the fractal coding method. The inner product is the important part in computing the Mean Square Error (MSE) between range-domain in matching processes with the eight symmetry transformations. The proposed algorithm adopted the developed Discreet Cosine Transform which is called fast DCT presented in [16] to minimize the computational cost of DCT for every domain and range block.

$\mathrm{Wu}$ [17] proposed a method of using regional search instead of whole image search. This method reduced the encoding time depending on the concept that the particular block has a high similarity to the blocks around and thus the time for matching will be minimized. This method minimized the time comparing to the conventional FIC.

Tong and $\mathrm{Pi}$ [18] presented a distinguished performance for the fractal coding by suggesting an adaptive search based on an efficient and simple condition for blocks similarity with a low reduction in compression quality. This condition depends on the standard deviation of the domain and range blocks as a filter for the largely nominated domain blocks. In addition, they replaced the conventional affine parameter, luminance offset with the range block mean.

$\mathrm{Wu}$ et al. [19] proposed a classification based on block variance. This classification reduces the consuming time of the fractal encoding process up to 480 times in comparison with the traditional one with a decrease in quality around $0.3 \mathrm{~dB}$. This reduction is because of the fact that each range blocks matches with domain blocks within the same class. In addition, they used Partial Distance Search (PDS) which was proposed in [20] with simplified of the eight domain block transformations.

Geroge [21] presented an improvement for speeding up the matching process for FIC using moments indexing block and stopping condition. A further suggestion to increase the compression ratio and speeding is presented by the author through applying the additional type of filtering and partitioning schema. This technique speeds up the encoding time 10 times without significant changes in the image quality.

$\mathrm{Wu}$ et al. [18] presented an enhancement to the search algorithm by proposing three methods: domain Intelligent Classification Algorithm (ICA), De-Redundancy Method (DRM) and Search Number Adaptive Control (SNAC). ICA is used to group the domain block with similar STD, DRM eliminates the domain blocks from domain pool which have very similar STD and SNAC ensures that range block with higher STD value matches with large domain blocks.

Speeding up fractal colored image compression is proposed by Al-Hilo and George [22]. They adopt $(\mathrm{Y}, \mathrm{U}, \mathrm{V})$ component instead of $(\mathrm{R}, \mathrm{G}, \mathrm{B})$ with 24-bits/pixel resolution to increase the compression rate and moment features as a descriptor for range and domain blocks to speeding up the fractal encoding process. The presented speed is about $96 \%$ higher compared with traditional FIC.

Another classification is produced by Kovacs [23] using two parameters which are: Approximate First Derivative (AFD) and Normalized Root Mean Square Error (NRMS). These parameters are used as a similarity measure between image blocks.

George and Al-Hilo [24] presented an enhancement to the previous method proposed in [22], in which the compression ratio is increased by $3 \%$ by using DPCM and shift coding for encoding the range mean and scale parameters. In addition, this method decreases encoding time by $66 \%$ and enhances the PSNR at around $5.3 \%$.

George and Al-Hilo [25] also proposed a speed up method in which it reduced the number of symmetry transformation of the domain block from 8 to 1 using first-order centralized moments with the predictor. This predictor gives the required block transform to get the best range-domain matching and thus the encoding time is reduced. This method show speed up encoding process around 7 times in comparison with conventional method with preserving compression ratio and PSNR.

Wang et al. [26] presented a fast FC approach by introduces correlation information feature to make the range-domain block matching near neighbor in the space. This feature results in a better result in term of reconstructed image quality with preserving the encoding time and compression ratio equal to other fast fractal coding studies.

Hasan and $\mathrm{Wu}$ [27] proposed Adaptive Fractal Image Compression algorithm AFIC based on several techniques to minimize the complexity of the matching process. These techniques are Adaptive Quadtree Partitioning Technique (AQPT), Zero Mean Intensity Level (ZMIL), Reducing the Domain Image Size (RDIS), Range Exclusion (RE) and Variance Domain Selection (VDS). These techniques work together to increase the compression ratio and decrease the encoding time at the same time. This algorithm showed minimize in time in the expense of a decrease in reconstructed image quality in comparison with several related studies.

Wang and Zheng [28] proposed a new FIC schema in which the Absolute value of Pearson's Correlation Coefficient (APCC) is used to classify the range and domain block. In addition, they sorted the domain blocks into sets according to the APCC to speed up the matching process between range and domain blocks. 
Fast fractal encoding algorithm based on Standard Deviation (STD) and Discrete Cosine Transform (DCT) are suggested by Wang et al. [6] to limit the search in the domain pool. They also used Auxiliary Encoding Algorithm (AEA) to improve the quality of the retrieved image when the range block size is large. They compared their proposed algorithm with the full search FIC to check the algorithm performance.

Pearson's correlation coefficient was also adopted in 2015 with the FIC in a method by Valarmathi et al. [13] to classify the domain blocks into three classes.

In addition, the iteration-free method proposed by [29] is employed in this method. This method is applied to grayscale images.

Jaferzadeh et al. [30] proposed an approach based on local binary features, intelligent STD threshold, and Humming distance techniques. This approach decreases the FIC encoding time comparing with full search method and decays in PSNR value of the reconstructed image.

\subsection{Fractal Audio Compression}

Regarding the FAC, it has been firstly discussed by Van and Axel in 1995 [31]. Then lately, Wannamaker and Vrscay [32] in 1997 utilized Wavelet Transform with the FC to obtain audio compression. They used the hybrid method between FC and WT since the audio signals have more smoothness than images. Their approach achieved a 6:1 compression ratio with good signal reconstruction fidelity.

In 2005, Xiao [12] presented the implementation and discussion of the fractal coding on an audio signal in his master thesis. He made empirical experiments to test the effect of this technique on another type of data instead of images. This study concluded that the fractal model is not appropriate to apply alone with audio signals and require some improvement techniques to apply with. In addition, the performance controlled by several parameters such as domain creation, range partitioning, bit allocation, and others. The achieved compression ratio in this study is around 3 to 6 and it uncovered that the preprocessing could be beneficial for more compression ratio. However, it did not discuss the fractal encoding time. George and Salih [33] proposed an audio compression system using FC based on PIFS and affine transformation using a PCM wave file. They showed the relation of block size and jumping step among encoding time, PSNR, MSE and compression ratio. In addition, they highlighted that the IFS coefficients (scale and offset) have a great effect on the compression ratio when using quantization and have not on the encoding time.

Potdar et al. [34] applied the fractal coding on the heart sound signal for compression. The schema used Variable Fractal Dimension (VFD) to classify the domain blocks. The performance result of the proposed schema exhibits relatively high encoding time with the particular sound sizes and good IPSNR value.

Ali et al. [35] proposed an approach to speeding up the FAC. They adopted a technique that the audio signal is divided into sub-signals and applied FC separately utilizing the parallel algorithm. In addition, they used a stop condition to stop matching process depends on the specific threshold.

Baden et al. [36] presented a FAC approach by adopting filtering method using moment descriptors to minimize the domain pool and speed up the matching process. The approach uses first and third order moment for classification.

Tabel 1. Summary of Literature Review

\begin{tabular}{lll}
\hline \multicolumn{1}{c}{ Article } & \multicolumn{1}{c}{ Method } & Domain \\
\hline Truong et al. 2000 & DCT \\
Wu 2000 & Regional search \\
Tong and Pi 2001 & Standard deviation and PDS \\
Wu et al. 2003 & Variance and mean \\
George 2005 & Block indexing using moments \\
Wu 2005 & ICA, DRM, and SNAC \\
Al-Hilo and George 2008 & Moment feature with (YUV) image components \\
Kovács 2008 & AFD and NRMS \\
George and Al-Hilo 2009 & Moment feature and DPCM and shift coding \\
George and Al-Hilo 2009 & First order centralized moment with predictor \\
Wan et al. 2010 & Correlation information feature \\
Hasan and Wu 2013 & AQPT, ZMIL, RDIS, RE and VDS \\
Wang and Zheng 2013 & APCC \\
Wang et al. 2013 & STD and DCT \\
Valarmathi 2015 & PCC with iteration-free method \\
Jaferzadeh 2016 & Local features, Intelligent STD and humming distance & Image \\
Wannamaker and Vrscay 1997 & Wavelet transform \\
Xiao 2005 & Traditional FC \\
George and Salih 2010 & Traditional FC \\
Potdar et al. 2012 & VFD \\
Ali et al. 2013 & Separating and stopping condition \\
Bedan and George 2013 & First and third order moment \\
\hline
\end{tabular}




\section{DISCUSSION}

As noticed in the literature review, most of the studies are dedicated to using fractal coding as a compressor with images, since fractal coding was firstly and mainly studied and introduced with the images. From a large number of studies that were proposed on fractal image coding, it has been demonstrated that fractal coding can successively apply with acceptable reconstruction image result. The future aspect of fractals seems to focus on adopting other techniques or transform to enhance the performance of the FIC. Built upon the success of FIC, this method has also been practiced in fractal audio coding which suggests the possibility of adopting FC in audio signals. A limited number of studies has been presented in the literature to investigate the efficiency of the FAC. Pre-processing can play a promising role in terms of improvement. A preprocessing step can be used to break the continuity in audio sequences, which may provide a way of compensating for the shortcomings of fractal systems. In fractal image coding, many applications have been applied with FIC as wavelets and DCT. We expect that the same concept may be applicable in fractal audio coding and could be equally successful. Concerning audio data, classification could accelerate the compression ratio and speed up FAC, although, classification is a more general topic that can be addressed from variant perspectives.

\section{CONCLUSION}

A review on the fractal image and audio compression, and their enhancing techniques have been presented in this study. It is obvious that interest is directed to fractal image more than the audio domain. The adaptation of fractal image coding is due to several reasons and the natural similarity in images is one of them. FIC produces high compression ratio in comparison to other image compression techniques. On the other hand, this study reveals the limitation of published articles in term of fractal coding in audio signals. The earlier studies in this field exhibit the ability to get an acceptable result by adopting fractal theory with audio. Further research is required in the area of fractal audio compression specifically and in fractal image compression generally in order to make these two techniques stand side by side with the counterpart.

\section{REFERENCES}

[1] N. A. Khairi, A. B. Jambek, and R. C. Ismail, "Performance evaluation of arithmetic coding data compression for internet of things applications," Indonesian Journal of Electrical Engineering and Computer Science, vol. 13, pp. 591-597, 2019.

[2] R. Ahmed, M. S. Islam, and J. Uddin, "Optimizing Apple Lossless Audio Codec Algorithm using NVIDIA CUDA Architecture," International Journal of Electrical and Computer Engineering (IJECE), vol. 8, pp. 70-75, 2018.

[3] K. Sayood, Introduction to data compression Fourth Edition: Elsevier, 2012.

[4] S. D. Kamble, N. V. Thakur, L. G. Malik, and P. R. Bajaj, "Color Video Compression Based on Fractal Coding Using Quadtree Weighted Finite Automata," in Information Systems Design and Intelligent Applications, ed: Springer, 2015, pp. 649-658.

[5] R. Chourasiya and A. Shrivastava, "A Study of image compression based transmission algorithm Using SPIHT for low bit rate application," Bulletin of Electrical Engineering and Informatics, vol. 2, pp. 117-122, 2013.

[6] X. Wang, D. Zhang, and X. Guo, "Novel hybrid fractal image encoding algorithm using standard deviation and DCT coefficients," Nonlinear Dynamics, vol. 73, pp. 347-355, 2013.

[7] A. Mammeri, B. Hadjou, and A. Khoumsi, "A survey of image compression algorithms for visual sensor networks," ISRN Sensor Networks, vol. 2012, 2012.

[8] T. Sheltami, M. Musaddiq, and E. Shakshuki, "Data compression techniques in Wireless Sensor Networks," Future Generation Computer Systems, vol. 64, pp. 151-162, 2016.

[9] N. K. M. S. AH, "A survey on fractal image compression key issues," Information Technology Journal, vol. 7, pp. 1085-1095, 2008.

[10] D. S. Seeli and M. Jeyakumar, "A study on fractal image compression using soft computing techniques," International Journal of Computer Science Issues(IJCSI), vol. 9, 2012.

[11] M.-S. Wu, "Genetic algorithm based on discrete wavelet transformation for fractal image compression," Journal of Visual Communication and Image Representation, vol. 25, pp. 1835-1841, 2014.

[12] H. Xiao, "Fractal Audio Coding," Master's Thesis, Queen's University, Canada, vol. 7, pp. 20-25, 2005.

[13] M. Valarmathi, M. Sobia, and R. B. Devi, "Iteration-Free Fractal Image Compression Using Pearson's Correlation Coefficient-Based Classification," in Informatics and Communication Technologies for Societal Development, ed: Springer, 2015, pp. 157-166.

[14] D. Liu and P. K. Jimack, "A survey of parallel algorithms for fractal image compression," Journal of Algorithms \& Computational Technology, vol. 1, pp. 171-186, 2007.

[15] T.-K. Truong, J.-H. Jeng, I. S. Reed, P. Lee, and A. Q. Li, "A fast encoding algorithm for fractal image compression using the DCT inner product," IEEE transactions on Image Processing, vol. 9, pp. 529-535, 2000.

[16] E. Feig and S. Winograd, "Fast algorithms for the discrete cosine transform," IEEE Transactions on Signal processing, vol. 40, pp. 2174-2193, 1992. 
[17] P.-Y. Wu, "Fast fractal image compression," in Information Technology: Coding and Computing, 2000. Proceedings. International Conference on, 2000, pp. 54-59.

[18] C. S. Tong and M. Pi, "Fast fractal image encoding based on adaptive search," IEEE Transactions on Image Processing, vol. 10, pp. 1269-1277, 2001.

[19] Y.-G. Wu, M.-Z. Huang, and Y.-L. Wen, "Fractal image compression with variance and mean," in Multimedia and Expo, 2003. ICME'03. Proceedings. 2003 International Conference on, 2003, pp. I-353-6 vol. 1.

[20] B. Chang-Da and R. M. Gray, "An improvement of the minimum distortion encoding algorithm for vector quantization," IEEE Transactions on Communications, vol. 33, pp. 1121-1133, 1985.

[21] L. E. George, "IFS coding for zero-mean image blocks," Iraqi Journal of Science, vol. 47, pp. 190-194, 2005.

[22] E. Al-Hilo and L. E. George, "Speeding-up Fractal Colored Image Compression using Moments Features," in Digital Image Computing: Techniques and Applications (DICTA), 2008, 2008, pp. 486-490.

[23] T. Kovács, "A fast classification based method for fractal image encoding," Image and Vision Computing, vol. 26, pp. 1129-1136, 2008.

[24] L. E. George and E. Al-Hilo, "Fractal Color Image Compression by Adaptive Zero-Mean Method," in 2009 International Conference on Computer Technology and Development, 2009, pp. 525-529.

[25] L. E. George and E. Al-Hilo, "Speeding-Up Color FIC Using Isometric Process Based on Moment Predictor," in Future Computer and Communication, 2009. ICFCC 2009. International Conference on, 2009, pp. 607-611.

[26] Q. Wang, D. Liang, and S. Bi, "Fast fractal image encoding based on correlation information feature," in Image and Signal Processing (CISP), 2010 3rd International Congress on, 2010, pp. 540-543.

[27] T. M. Hasan and X. Wu, "An adaptive fractal image compression," 2013.

[28] J. Wang and N. Zheng, "A novel fractal image compression scheme with block classification and sorting based on Pearson's correlation coefficient," IEEE transactions on image processing, vol. 22, pp. 3690-3702, 2013.

[29] H. T. Chang and C. J. Kuo, "Iteration-free fractal image coding based on efficient domain pool design," IEEE Transactions on Image Processing, vol. 9, pp. 329-339, 2000.

[30] K. Jaferzadeh, I. Moon, and S. Gholami, "Enhancing fractal image compression speed using local features for reducing search space," Pattern Analysis and Applications, pp. 1-10, 2016.

[31] A. Van de Walle, "Relating fractal image compression to transform methods," Citeseer, 1995.

[32] R. A. Wannamaker and E. R. Vrscay, "Fractal wavelet compression of audio signals," Journal of the Audio Engineering Society, vol. 45, pp. 540-553, 1997.

[33] L. E. George and H. M. Salih, "Audio Compression Using Fractal Coding," Diyala Journal for Pure Sciences (DJPS), vol. 6, pp. 61-70, 2010.

[34] R. M. Potdar, M. K. Kowar, A. Biswas, and M. Amtey, "Multi-Scale Domain Classification Based Heart Sound Compression," International Journal of Soft Computing and Engineering (IJSCE), vol. 2, pp. 41-44, 2012.

[35] S. M. Ali, L. E. George, and H. B. Taher, "Speeding up Audio Fractal Compression," International Journal of Advanced Research in Computer Science and Software Engineering, vol. 3, pp. 86-92, 2013.

[36] A. K. Bedan and L. E. George, "Speeding-up Fractal Audio Compression Using Moment Descriptors," International Journal of Scientific \& Engineering Research, vol. 4, 2013. 